\title{
Hemolytic Uremic Syndrome-associated Encephalopathy Successfully Treated with Corticosteroids
}

\author{
Takashi Hosaka, Kiyotaka Nakamagoe and Akira Tamaoka
}

\begin{abstract}
:
The encephalopathy that occurs in association with hemolytic uremic syndrome (HUS), which is caused by enterohemorrhagic Escherichia coli (E. coli), has a high mortality rate and patients sometimes present sequelae. We herein describe the case of a 20-year-old woman who developed encephalopathy during the convalescent stage of HUS caused by E. coli O26. Hyperintense lesions were detected in the pons, basal ganglia, and cortex on diffusion-weighted brain MRI. From the onset of HUS encephalopathy, we treated the patient with methylprednisolone (mPSL) pulse therapy alone. Her condition improved, and she did not present sequelae. Our study shows that corticosteroids appear to be effective for the treatment of some patients with HUS encephalopathy.
\end{abstract}

Key words: enterohemorrhagic Escherichia coli O26, hemolytic uremic syndrome, encephalopathy, methylprednisolone pulse, diffusion-weighted imaging, magnetic resonance imaging

(Intern Med 56: 2937-2941, 2017)

(DOI: 10.2169/internalmedicine.8341-16)

\section{Introduction}

Hemolytic uremic syndrome (HUS) is defined as a triad of hemolytic anemia with schistocytes, thrombocytopenia, and acute kidney failure $(1,2)$. In Japan, HUS develops in approximately $3.2-4.0 \%$ of all patients with symptomatic enterohemorrhagic Escherichia coli (EHEC) (3). Neurological complications such as stroke, seizure, coma, and hemiparesis occur in approximately $10-50 \%$ of cases $(4,5)$. Complications, leading to death in $17 \%$ and severe sequelae in $23 \%$ of patients, are the main causes of acute mortality $(2,6)$. Although it has been reported that methylprednisolone (mPSL) pulse therapy improves the poor prognosis (7), early plasma exchange was shown to ameliorate HUS encephalopathy (8) and eculizumab (a monoclonal antibody against complement factor C5) was found to be effective against intractable HUS (9); however, these studies only involved a small number of patients; thus, there is no specific treatment available for treating neurological complications.

In this report, we present the case of a 20-year-old woman with coma after the onset of HUS caused by EHEC O26. Due to her symptoms and abnormalities on diffusion- weighted magnetic resonance imaging (DW-MRI) of her brain, we diagnosed the patient with HUS encephalopathy and treated her with mPSL pulse therapy alone. Her symptoms improved with mPSL pulse therapy, and she showed no neurological sequelae. This is the first report of mPSL pulse therapy being effective when administered alone immediately after the onset of HUS encephalopathy; thus, it is important to develop methods for diagnosing HUS encephalopathy in the early stage and to investigate therapies for HUS encephalopathy.

\section{Case Report}

The patient was a 20 -year-old woman. She was observed with lower left abdominal pain and diarrhea, followed by high fever; she also developed bloody diarrhea. Four days after the appearance of the symptoms of gastroenteritis, she was admitted to a nearby hospital and was treated with hydration and antibiotics (Fosfomycin). Her digestive symptoms improved after treatment; however, she developed hemolytic anemia and acute kidney injury five days after the appearance of the symptoms of gastroenteritis. Her stool culture revealed EHEC serotype O26, and the EHEC:O26 

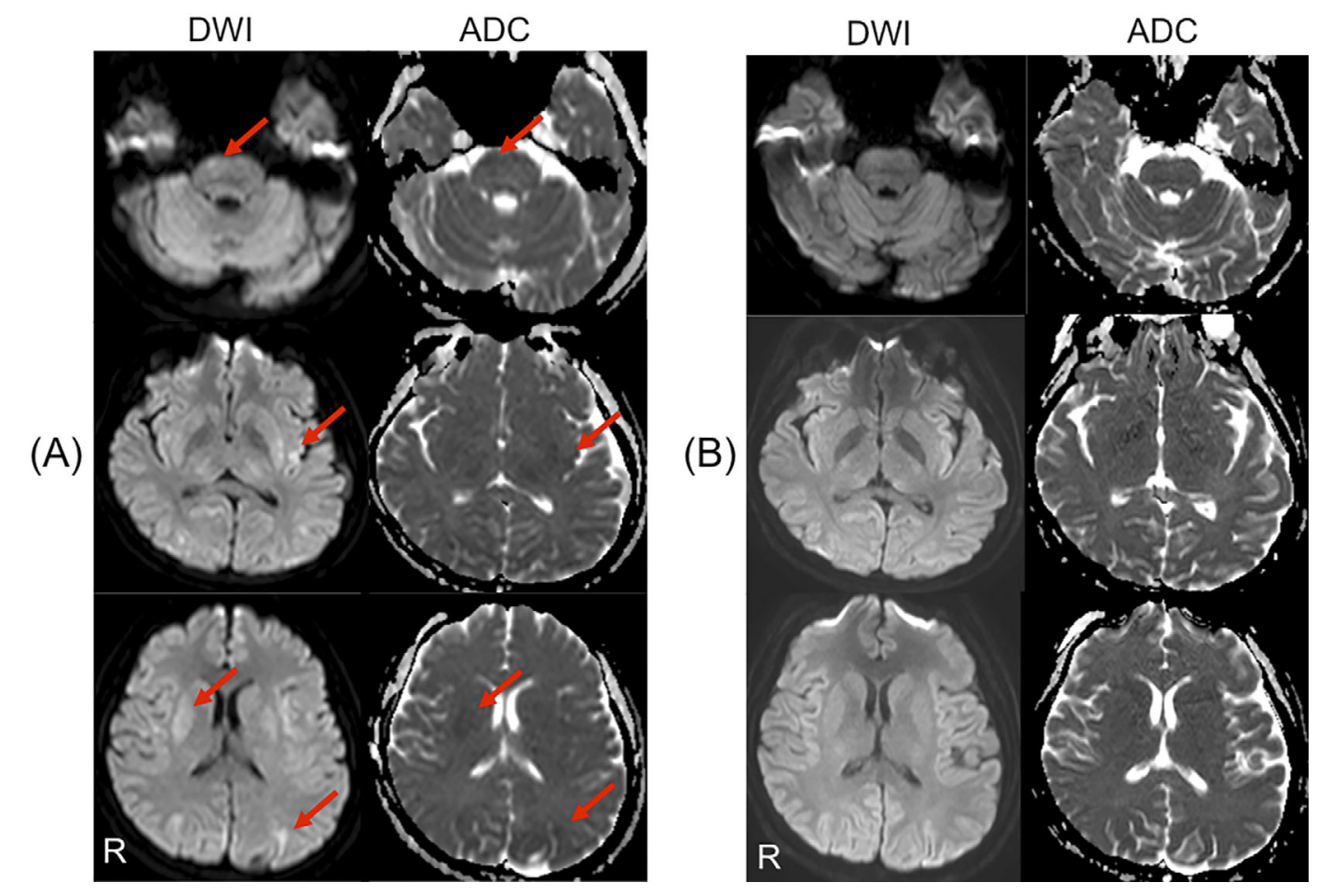

Figure. Brain MRI. Brain MRI [axial view of diffusion-weighted imaging (DWI) and the apparent diffusion coefficient (ADC)]. (A) Brain MRI [FLAIR (TR/TE=11,000.0/140.0 ms), DWI, and ADC $\left.\left(b=1,000 \mathrm{~s} / \mathrm{mm}^{2}, T R / T E=3,500.0 / 67.1 \mathrm{~ms}\right)\right]$ on admission. Hyperintense lesions were detected in the right ventral pons, left insular cortex, bilateral basal ganglia, and left occipital cortex were on DWI (arrows). Some of these lesions showed decreased ADC values (arrows). (B) Brain MRI [FLAIR (TR/ TE=11,000.0/125.0 ms), DWI, and ADC ( $b=1,000 \mathrm{~s} / \mathrm{mm}^{2}$, TR/TE=5,000.0/65.0 ms $\left.)\right]$ after methylprednisolone pulse therapy. The DWI and ADC values abnormalities that were present on admission had disappeared.

was a verotoxin-producing $E$. coli; she was therefore diagnosed with HUS caused by EHEC:O26. She was treated with supportive care, and her symptoms improved; however, she presented with a sudden disturbance of consciousness 10 days after the appearance of gastroenteritis symptoms. The disturbance of consciousness was prolonged and the patient was transferred to our hospital on the same day.

The patient had no particular past or family history. She lived alone and did not eat raw food. No one around her presented similar symptoms. She was observed to have high fever, tachycardia, anemia of the palpebral conjunctiva, and pedal edema; however, she did not have hypertension.

Neurological findings demonstrated that she was in a coma [Glasgow Coma Scale (GCS) E2V1M1] and had neck stiffness. Her left eye became internally dislocated-neither eye could be abducted-and she was negative for the doll's eye reflex. She also had ocular bobbing. Anisocoria was not noted, and the light reflex was normal; however, the eyelash reflex was absent. Her left arm was flexed, and her other extremities showed flaccid paresis. Her tendon reflexes were normal, and she did not show pathological reflexes or involuntary movement.

Laboratory tests revealed C-reactive protein elevation $(0.79 \mathrm{mg} / \mathrm{dL})$, hemolytic anemia (schistocytes $0.69 \%$ ), a low platelet count $\left(6.1 \times 10^{4} / \mu \mathrm{L}\right)$, liver dysfunction [aspartate aminotransferase (AST) $153 \mathrm{IU} / \mathrm{L}$, alanine aminotransferase (ALT) $235 \mathrm{IU} / \mathrm{L}$ ], and renal dysfunction (creatinine $1.13 \mathrm{mg}$ / dL), but not electrolyte abnormality. The activity of disintegrin-like plasma and metalloproteinase with thrombospondin type 1 motifs 13 (ADAMTS13) was mildly reduced $(64.7 \%)$, and the patient was negative for ADAMTS 13 inhibitors. Thus, thrombotic thrombocytopenic purpura was ruled out. A stool culture revealed EHEC O26. In the cerebrospinal fluid (CSF), the cell count and protein concentration were within the normal ranges; however, the level of interleukin (IL)-6 was elevated $(229 \mathrm{pg} / \mathrm{mL}$; normal range < $4 \mathrm{pg} / \mathrm{mL})$.

Hyperintense lesions were detected in the right ventral pons, left insular cortex, bilateral basal ganglia, and left occipital cortex on DW-MRI of the brain (Figure A), and some of these lesions showed decreased apparent diffusion coefficient (ADC) values. N-isopropyl-p-[(123)I]-iodoamphetamine single photon emission computed tomography revealed the reduction of the cerebral blood flow in the orbitofrontal area, the cerebellum, and the brainstem. There were also diffuse bursts of slow waves on an electroencephalogram; however, the wave was not spiked or sharp.

Due to the sudden disturbance of consciousness after the onset of HUS and the abnormality of her bilateral deep gray matter on brain MRI, we diagnosed the patient with HUS 
Table. A Review of the Patients with EHEC-associated HUS Encephalopathy who Underwent mPSL Pulse Therapy.

\begin{tabular}{|c|c|c|c|c|c|c|}
\hline $\begin{array}{c}\text { Reference } \\
\text { No. }\end{array}$ & Age/Sex & $\begin{array}{l}\text { Days from onset } \\
\text { of HUS to } \\
\text { encephalopathy }\end{array}$ & $\begin{array}{l}\text { Days from onset of } \\
\text { HUS encephalopathy } \\
\text { to mPSL pulse therapy }\end{array}$ & $\begin{array}{l}\text { EHEC } \\
\text { serotype }\end{array}$ & $\begin{array}{c}\text { Additional } \\
\text { anti-proinflammatory } \\
\text { cytokine therapy }\end{array}$ & $\begin{array}{l}\text { Outcome } \\
\text { (sequelae) }\end{array}$ \\
\hline 19 & $7 / \mathrm{M}$ & A few days & 0 days & $\mathrm{O} 157$ & IVIg & Alive (no) \\
\hline 20 & $28 / \mathrm{F}$ & 5 days & 1 day & O157 & $\gamma$-globulin, PE & Alive (no) \\
\hline 21 & $7 / \mathrm{F}$ & 2 days & a few days & O111 & PDF, IVIg & Alive (no) \\
\hline \multirow[t]{12}{*}{7} & $16-19$ years/F & 8 days & 4 days & O111 & PE, PMX, IVIg & Alive (no) \\
\hline & $<2$ years/M & 7 days & 6 days & O111 & PMX, IVIg & Alive (no) \\
\hline & $20-29$ years/F & 8 days & 3 days & O111 & PE, PMX & $\begin{array}{l}\text { Alive (intellectual } \\
\text { disability) }\end{array}$ \\
\hline & $5-9$ years/F & 7 days & 2 days & O111 & IVIg & Alive (no) \\
\hline & $20-29$ years/F & 8 days & 2 days & $\mathrm{O} 111$ & PE, PMX & Alive (no) \\
\hline & 10-15 years/M & 8 days & 1 day & O111 & IVIg & Alive (no) \\
\hline & 5-9 years/F & 9 days & less than 1 day & O111 & PE & Alive (no) \\
\hline & $16-19$ years/F & 10 days & 1 day & O111 & IVIg & Alive (no) \\
\hline & $10-15$ years/F & 9 days & 0 days & O111 & PE, PMX, IVIg & Alive (no) \\
\hline & $20-29$ years/F & 10 days & 2 days & O111 & PE, IVIg & Alive (no) \\
\hline & $5-9$ years/F & 11 days & less than 1 day & O111 & PE, IVIg & Alive (no) \\
\hline & $16-19$ years/F & 16 days & less than 3 days & $\mathrm{O} 111$ & PE, PMX & Alive (no) \\
\hline 22 & $26 / \mathrm{F}$ & 0 days & 6 days & O157/O111 & $\begin{array}{l}\text { PE, Cytokine } \\
\text { adsorption }\end{array}$ & $\begin{array}{c}\text { Alive (higher } \\
\text { brain dysfunction) }\end{array}$ \\
\hline 23 & $14 / \mathrm{F}$ & 2 days & 0 days & O111 & $\mathrm{PE}$ & Alive (no) \\
\hline Present case & $20 / \mathrm{F}$ & 4 days & 0 days & $\mathrm{O} 26$ & None & Alive (no) \\
\hline
\end{tabular}

M: male, F: female, HUS: hemolytic uremic syndrome, EHEC: enterohemorrhagic Escherichia coli, mPSL: methylprednisolone, IVIg: IV immunoglobulin, PE: plasma exchange, PDF: plasma diafiltration, PMX: polymyxin B-immobilized column direct hemoperfusion

encephalopathy. We treated her with mPSL pulse therapy $(1,000 \mathrm{mg} /$ day for three days) alone, soon after she was transferred to our hospital (10 days after the appearance of the symptoms of gastroenteritis). Following treatment, her consciousness disturbance improved (GCS E4V5M6), at 14 days after the appearance of the symptoms of gastroenteritis. The patient's ocular bobbing disappeared, and her hemolytic anemia, low platelet count, and renal dysfunction improved. Diffusion weighted image (DWI) and ADC abnormalities were not detected on brain MRI after mPSL pulse therapy (Figure B). A second three-day course of mPSL pulse therapy was administered 17 days after the appearance of the symptoms of gastroenteritis. The patient was discharged without sequelae 33 days after the appearance of the symptoms of gastroenteritis.

\section{Discussion}

As HUS encephalopathy is a major cause of acute mortality, it is necessary to diagnose and treat the condition as soon as possible. Diffuse brain edema and signal changes in the bilateral deep gray matter, which participates in the lateral segment of the lenticular nucleus, can be detected in patients who have severe symptoms. Increased DWI signals are detected in the thalamus, cerebellum, and brain stem (10). In the earliest phase, abnormalities are not detected on fluid-attenuated inversion recovery (FLAIR) imaging or T2-weighted imaging (T2WI); however, DWI abnormalities are detected in cases such as ours $(11,12)$. Thus, it is very important to diagnose HUS encephalopathy in the earliest phase by conducting DWI when performing brain MRI.

In our case, DWI showed restricted diffusion, and some of these lesions showed decreased ADC values. The main neuropathological observations in autopsy cases were diffuse brain edema, astrogliosis, and microgliosis, which were prominent in the thalamus and pons; however, signs of thrombosis were nonexistent, and signs of microbleeding around vessels were rare (13). These lesions were transient and reversible. We therefore thought that they did not represent ischemic changes. It has been reported that some patients with multiple sclerosis or mild encephalitis/encephalopathy with reversible splenial lesions showed hyperintensity on DWI and decreased ADC values-this was true in our case $(14,15)$. The mechanisms underlying the transient reduction of diffusion in patients with these inflammatory diseases may be related to intramyelinic edema, interstitial edema, and the infiltration of inflammatory cells $(14,16)$. Thus, the DWI and ADC abnormalities may be related to these mechanisms.

It is thought that HUS encephalopathy occurs due to direct damage to the endothelium from verotoxin and neuroinflammation due to the release of cytokines, including IL-6 and tumor necrosis factor (TNF)- $\alpha(17,18)$. Actually, in our case, the level of IL-6 was elevated in the CSF. Thus, it was considered that treatment with mPSL pulse therapy may have been effective.

mPSL pulse therapy should be considered for patients with severe symptoms; however, the efficacy of mPSL pulse therapy for HUS encephalopathy has not yet been estab- 
lished. We investigated the cases in which HUS encephalopathy was treated with mPSL pulse therapy (Table) $(7,19-23)$. The number of days from the onset of HUS to encephalopathy and mPSL pulse therapy varied. With the exception of one patient, the patients treated with mPSL pulse therapy had good outcomes without sequelae. It has been reported that because mPSL suppresses inflammatory cytokines, mPSL pulse therapy is effective for patients with HUS encephalopathy and improves the poor prognosis (7). We showed-once more-that HUS encephalopathy is related to neuroinflammation and that treatment with mPSL therapy is important. We first treated our patient with mPSL pulse therapy. Although we considered plasma exchange as the next step, mPSL pulse therapy was effective, and the patient did not receive any other therapies. We administered mPSL pulse therapy twice. Our case was the only case in which mPSL pulse therapy was administered alone; all of the other cases involved combined therapies, such as plasma exchange, IV immunoglobulin, plasma diafiltration, and/or polymyxin B-immobilized fiber column direct hemoperfusion. Our case was very interesting and important because the patient was treated with mPSL pulse therapy alone and had a good outcome without sequelae. It has been reported that oral steroids ( $5 \mathrm{mg} / \mathrm{kg} /$ day) were not able to hematologically or neurologically modify the acute phase of HUS encephalopathy in children in comparison to a placebo (24). However, the discrepancy may not only be caused by differences in children and adults but also by the dose of steroids or the method of administration. Thus, high doses of mPSL may be effective for treating some patients with HUS encephalopathy.

In conclusion, our study shows that corticosteroids appear to be effective for the treatment of some patients with HUS encephalopathy. Our finding indicate that HUS encephalopathy can be treated with mPSL pulse therapy alone when neurological complications are reversible if HUS encephalopathy is diagnosed in the earliest phase. If additional study is advanced based on the findings of this case report, the diagnosis of HUS encephalopathy will be improved.

The authors state that they have no Conflict of Interest (COI).

\section{Financial Support}

This work was supported by JSPS KAKENHI Grant Number JP 26460901.

\section{References}

1. Igarashi T, Ito S, Sako M, et al. Guidelines for the management and investigation of hemolytic uremic syndrome. Clin Exp Nephrol 18: 525-557, 2014.

2. Trachtman H, Austin C, Lewinski M, et al. Renal and neurological involvement in typical Shiga toxin-associated HUS. Nat Rev Nephrol 8: 658-669, 2012.

3. Yahata Y, Misaki T, Ishida Y, et al. Epidemiological analysis of a large enterohaemorrhagic Escherichia coli O111 outbreak in Japan associated with hemolytic uremic syndrome and acute encephalo- pathy. Epidemiol Infect 143: 2721-2732, 2015.

4. Saini A, Emke AR, Silva MC, et al. Response to eculizumab in Escherichia coli O157: H7-induced hemolytic uremic syndrome with severe neurological manifestations. Clin Pediatr (Phila) 54: 387-389, 2015.

5. Kamioka I, Yoshiya K, Satomura K, et al. Risk factors for developing severe clinical course in HUS patients: a national survey in Japan. Pediatr Int 50: 441-446, 2008.

6. Nathanson S, Kwon T, Elmaleh M, et al. Acute neurological involvement in diarrhea-associated hemolytic uremic syndrome. Clin J Am Soc Nephrol 5: 1218-1228, 2010.

7. Takanashi J, Taneichi H, Misaki T, et al. Clinical and radiologic features of encephalopathy during 2011 E. coli O111 outbreak in Japan. Neurology 82: 564-572, 2014.

8. Colic E, Dieperink H, Titlestad K, Tepel M. Management of an acute outbreak of diarrhoea-associated haemolytic uraemic syndrome with early plasma exchange in adults from southern Denmark: an observational study. Lancet 378: 1089-1093, 2011.

9. Lapeyraque AL, Malina M, Fremeaux-Bacchi V. Eculizumab in severe Shiga-toxin-associated HUS. N Engl J Med 364: 2561-2563, 2011.

10. Steinborn M, Leiz S, Rüdisser K, et al. CT and MRI in hemolytic uremic syndrome with central nervous system involvement: distribution of lesions and prognostic value of imaging findings. Pediatr Radiol 34: 805-810, 2004.

11. Löbel U, Eckert B, Simova O, et al. Cerebral magnetic resonance imaging findings in adults with hemolytic uremic syndrome following an infection with Escherichia coli, subtype O104:H4. Clin Neuroradiol 24: 111-119, 2014.

12. Donnerstag F, Ding X, Pape L, et al. Patterns in early diffusionweighted MRI in children with haemolytic uraemic syndrome and CNS involvement. Eur Radiol 22: 506-513, 2012.

13. Magnus T, Röther J, Simova $O$, et al. The neurological syndrome in adults during the 2011 northern German E. coli serotype O104: H4 outbreak. Brain 135: 1850-1859, 2012.

14. Inal M, Unal B, Kala I, et al. ADC evaluation of the corticospinal tract in multiple sclerosis. ActaNeurol Belg 115: 105-109, 2015.

15. Gawlitza M, Hoffmann KT, Lobsien D. Mild encephalitis/encephalopathy with reversible splenial and cerebellar lesions (MERS type II) in a patient with hemolytic uremic syndrome (HUS). J Neuroimaging 25: 145-146, 2015.

16. Osuka S, Imai H, Ishikawa E, et al. Mild encephalitis/encephalopathy with a reversible splenial lesion: evaluation by diffusion tensor imaging. Two case reports. Neurol Med Chir (Tokyo) 50: 1118-1122, 2010.

17. Schuppner R, Maehlmann J, Dirks M, et al. Neurological sequelae in adults after E. coli O104: $\mathrm{H} 4$ infection-induced hemolyticuremic syndrome. Medicine (Baltimore) 95: e2337, 2016.

18. Agarwal R, Davis C, Altinok D, et al. Posterior reversible encephalopathy and cerebral vasoconstriction in a patient with hemolytic uremic syndrome. Pediatr Neurol 50: 518-521, 2014.

19. Oki E, Tsuruga K, Tsugawa $K$, et al. Alternative treatment for systemic involvement in a child with postdiarrheal hemolytic-uremic syndrome. Clin Nephrol 70: 354-356, 2008.

20. Yoshimitsu M, Hayashi N, Kaneko Y, et al. An adult case of combined encephalopathy and hemolytic uremic syndrome caused by Escherichia coli O157. Nihon Shokakibyo Gakkai Zasshi 108: 7479, 2011 (in Japanese).

21. Shimizu M, Nakayama Y, Taniguchi T. Successful treatment of enterohemorrhagic Escherichia coli O111-induced acute encephalopathy and hemolytic-uremic syndrome with plasma diafiltration. Ther Apher Dial 18: 516-518, 2014.

22. Ito M, Shiozaki A, Shimizu M, et al. Hemolytic-uremic syndrome with acute encephalopathy in a pregnant woman infected with epidemic enterohemorrhagic Escherichia coli: characteristic brain images and cytokine profiles. Int J Infect Dis 34: 119-121, 2015. 
23. Yada N, Fujioka M, Bennett CL, et al. STEC:O111-HUS complicated by acute encephalopathy in a young girl was successfully treated with a set of hemodiafiltration, steroid pulse, and soluble thrombomodulin under plasma exchange. Clin Case Rep 3: 208212, 2015.

24. Perez N, Spizzirri F, Rahman R, Suarez A, Larrubia C, Lasarte P. Steroids in the hemolytic uremic syndrome. Pediatr Nephrol 12:
101-104, 1998.

The Internal Medicine is an Open Access article distributed under the Creative Commons Attribution-NonCommercial-NoDerivatives 4.0 International License. To view the details of this license, please visit (https://creativecommons.org/licenses/ by-nc-nd/4.0/).

(C) 2017 The Japanese Society of Internal Medicine Intern Med 56: 2937-2941, 2017 